CK2a_S_pombe

CK2a_Rattus

CK2a_H_sapiens

CK2a_T_cruzi

CK2a_Leishmania

CK2a_s_pombe

CK2a Rattus

CK2a_H_sapiens

CK2a T cruzi

CK2a_Lèishmania

CK2a_s_pombe

CK2a_Rattus

CK2a_H_sapiens

CK2a T cruzi

CK2a_Lèishmania

CK2a s pombe

CK2a_Rattus

CK2a_H_sapiens

CK2a_T_cruzi

CK2a_Lēishmania

CK2a_s pombe

CK2a_Rattus

CK2a H sapiens

CK2a_T_cruzi

CK2a Leishmania

CK2a_s_pombe

CK2a Rattus

CK2a_H_sapiens

CK2a T cruzi

CK2a_Leishmania

CK2a_s_pombe

CK2a_Rattus

CK2a_H_sapiens

CK2a T cruzi

CK2a_Lèishmania

CK2a_s pombe

CK2a_Rattus

CK2a H sapiens

CK2a_T cruzi

CK2a_Lèishmania

- - - - - - -

-

MTDQDSSGRII IVTRPVPASPRPPENNGDVSHVTTDCKSOKNTGENTVEAKRTLTENEIR

-MANVDAAAHS-ADGGRHGGGGGGSGDESKAK

\title{
ACTIVE SITE
}

AAPVVSVSRVYAHVNEEMPREYWDYENMQEVFGY-QDNYEI IRKVGRGKYSEVFEGLNVL SGPVPSRARVYTDVNTHRPREYWDYESHVVEWGN-ODDYOLVRKLGRGKYSEVFEAINIT SGPVPSRARVYTDVNTHRPREYWDYESHVVEWGN-QDDYQLVRKLGRGKYSEVFEAINIT HHETGVISHPFWFVNNNMP PSYWDYERMTIEYSS-GEPYELIOKIGRGKYSEVFRCRNRI KTKPEEYEHPFWYVYRQRGVSYWDYKNARVDFNANLAPYELLQKIGRGKYSEVFRGRNRN

\section{$*:::: *: * * * * * * * * *$.}

\section{ACTIVE SITE / CK2 $\beta$ INTERACTION SURFACE}

NNRKCI IKVLKPVKYKKI KRE I K ILQNLAGGPN I ISLLDIVRDPESKTP SL I FEFVDNID NNEKVVVK ILKPVKKKKI KRE I I IENLRGGPN I ITLAD IVKDPVSRTPALVFEHVNNTD NNEKVVVK ILKPVKKKKI KRE I K ILENLRGGPN I ITLADIVKDPVSRTPALVFEHVNNTD NGELCVLK ILKPVRLKKI HRE IS ILONLCGGPNVLRLLDVVS ISPEGTPVLVTENLEPAE NGCLCVLKLLKPVRYQKILREIS ILRNLCGGPNVVRLLDVLRDTESQTVVLVTEYVHNPT *, $:: *: * * * *: ; * * * * *, * *, * * * * * *: ; * *:: \quad * * * ;: ;$

ACTIVE SITE

-FRTL--YPTLSDYDIRYYLYELLKALDFCHSRGIMHRDVKPHNVI IVHKKRKLRLIDWG -FKQL--YQTLTDYDIRFYMYEILKALDYCHSMGIMHRDVKPHNVMIDHEHRKLRLIDWG -FKQL--YQTLTDYDIRFYMYEI LKALDYCHSMGIMHRDVKPHNVMIDHEHRKLRLIDWG SFRSLMNSGSLSNFDMRYYMYEVLRCLHFAHSHGIFHRDIKPHNI I IDHORRKLRIADWG TLRNLLYSNKLSNF DMRYYLYEI LRSLDFAHRRG IFHRDIKPYNVMIDHERK ILRVIDWG

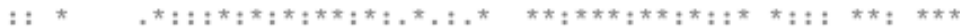
ACTIVE SITE I ACTIVATION LOOP

LAEFYHAGMEYNVRVASRYFKGPELLVDFREYDYSLD IVSFGVMFAALIFKKEPFFRGRD LAEFYHPGQEYNVRVASRYFKGPELLVDYQMYDYSLDMWSLGCMLASMIFRKEPFFHGHD LAEFYHPGQEYNVRVASRYFKGPELLVDYOMYDYSLDMWSLGCMLASMIFRKEPFFHGHD LGEYYIHGQAYNVCVGTRNFKAPELLLGLRLYDYSLD IWSVGCILAEMLFR IFPFFRGQN LGEYYIHGQALNCGVATRHYKGPELLVGYRHYDYSLD IWCLGCVLAGMLFRSDPFFVGAN

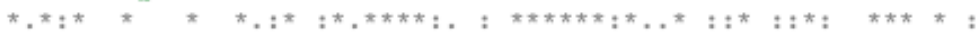

NYDQLVKIAKVLGTDELFAYVQKYQIVLDRQYDNILGQYP--KRDWY-FVN--RDNRSLA NYDQLVRIAKVLGTEDLYDYIDKYNI ELDPRFNDILGRHS--RKRWERFVH--SENOHLV NYDQLVRIAKVLGTEDLYDYIDKYNI ELDPRFNDILGRHS--RKRWERFVH--SENQHLV NEDQLYRILEVIGTEDLTRYARKYDISLPRFLFGSGGLF KRMKKPWY IF --VNDQCESWC NEDQLLQIVAVFGTKALYRYLDKYQCRISRVVESSMSALPDEHVDWRRYIKRGSVQESWC ***:**:**.* ***: : $:$ : $:$ : :

NDEAIDLLNRLLRYDHQERLTCQEAMAHPYFQVLK--- SPEALDFLDKLLRYDHOSRLTAREAMEHPYFYTVVKDQARMSSAGMAGGSTPVSSANMMS SPEALDFLDKLLRYDHQSRLTAREAMEHPYFYTVVKDQARMGSSSMPGGSTPVSSANMMS DVHAVDLLDKMLRLDHQER ILAWDAMQHPFFDP IRSALREDPQEQY PQ-- - - - - DATALDLLDKMLQFDHQDRIMAHEAMQHPFFAPVRDALARDSQEHYPVARR---------

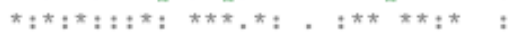

Figure S2: Multiple sequence alignment of CK2 $\alpha$ orthologous from the indicated species. Sequences were aligned using the ClustalW tool (https://www.genome.jp/tools-bin/clustalw). The conserved active site of the enzyme from those species is indicated. 